\title{
Delta Scuti Stars in Open Clusters: w2 and w20 in NGC 2264 and h501 and h906 in $\alpha$ Per
}

\author{
J. H. Peña ${ }^{1}$, R. Peniche ${ }^{1}$, F. Cervantes ${ }^{1,2}$, R. M. García ${ }^{1,2}$, \\ J. P. Sareyan ${ }^{3}$
}

\section{Motivation}

There are many advantages to studying variable stars in open clusters; the parameters that determine the evolution of the stars, such as chemical composition and age, can be considered to be the same for all the stars in the cluster. These data, along with the mass, effective temperature and superficial gravity, allow us to better determine the physics which explain the pulsation mechanisms. In the present paper the variable stars h501 and h906 in $\alpha$ Per and w2 and w20 in NGC 2264 are studied. In particular, the reasons why $\delta$ Scuti stars coexist with early type stars in NGC 2264 is examined.

\section{Observations}

The photometric data obtained is of two types: differential for the variables and absolute for the stars in the direction of the cluster. Both were secured at the Observatorio Astronomico Nacional, México. The data for NGC 2264 were acquired in the winter 1985-86, whereas that for $\alpha$ Per in autumn 1998. Membership in the cluster and physical characteristics of the observed stars were determined through Strömgren uvby $\beta$ photometry. These data were combined with that compiled from the literature for both clusters. For NGC 2264 a comparison of the photometric values obtained was made with those papers which presented absolute $U B V$ and Strömgren photometry. These sources are: Walker (1956) with $U B V$; Pérez et al. (1989); Neri, Chavarría, \& de Lara (1993), and Strom, Strom, \& Yost (1971) with Strömgren photometry. A final compilation of 160 stars was obtained. For $\alpha$ Per the sources which presented absolute photometry were: Heckmann, Dieckvoss, \& Kox (1956), Mitchell (1960) in $V$, and Crawford \& Barnes (1974) in Strömgren. The final compiled set consists of 150 stars.

\section{Results}

a) The clusters. The procedure followed has been described in Peña \& Peniche (1994). For NGC 2264, a histogram of the distances determined for the stars

\footnotetext{
${ }^{1}$ Instituto de Astronomía, UNAM, Ap. Postal 70-264, Mexico D. F. 04510, México

${ }^{2}$ Facultad de Ciencias, UNAM, Cd. Investigacion Mexico D. F. 04510, México

${ }^{3}$ Observatoire de la Cote d'Azur, CERGA, F-06130 Grasse, France
} 
in the direction of NGC 2264 was done for three different spectral types: B, $\mathrm{A}$ and $\mathrm{F}$. What was immediately evident was that the early type stars belong to an association at a distance between 600 and $800 \mathrm{pc}$, whereas the late type stars show a maximum at a closer distance, $350 \mathrm{pc}$. The $\delta$ Scuti stars belong to this latter group. For $\alpha$ Per the assigned membership is in agreement with the membership probability given by Heckmann, Dieckvoss, \& Kox (1956), Roman \& Morgan (1950) and Smart \& Ali (1940) from proper motion studies. The histogram of the distances determined for the stars in the direction of $\alpha$ Per shows only one clearly discernible cluster at $200 \mathrm{pc}$.

b) The $\delta$ Scuti Stars. Very little is known about these stars. Since the $\delta$ Scuti stars lie within the instability strip limits between A2 and F5, the prescriptions described above for clusters are also applicable. $M_{v}, \log T_{\text {eff }}, \log g$ have been calculated for each star, which, along with the periods, allowed the determination of the pulsational constant.

\section{Conclusions}

The most important contribution of this paper is the determination of membership of the variables to the clusters. In particular, for NGC 2264 the result is quite important in the sense that it is not a single cluster, but two in the same direction. The $\delta$ Scuti stars, then, are consistent with the evolutionary models for these type of stars.

Acknowledgments. Special thanks to the staff of the SPM observatory who made the observations possible. We would like to thank the funds provided by Conacyt through grant $3925 \mathrm{E}$ and an agreement with the CNRS, France. Typing and proofreading were done by J. Orta and J. Miller. We are indebted to the late T. Gómez for her assistance in the reduction of the differential photometric data. Two of us, FC and RMG, thank the Instituto de Astronomía, UNAM for the funds provided for the observing season which contributed to the development of our undergraduate bachelor's theses. This research has made use of the SIMBAD database operated at CDS, Strasbourg, France.

\section{References}

Crawford, D. L. \& Barnes, J. V. 1974, AJ, 79, 687

Heckmann, von O., Dieckvoss, W., \& Kox, H. 1956, Astron. Nach., 283, 109

Mitchell, R. I. 1960, ApJ, 132, 68

Neri, L., Chavarría-K., C. \& de Lara, E. 1993, A\&AS, 102, 201

Peña, J. H. \& Peniche, R. 1994, RevMexAA, 28, 139

Pérez, M. R., Joner, M. D., Thé, P. S., \& Westerlund, B. E. 1989, PASP, 101, 195

Roman, N. G. \& Morgan, W. W. 1950, ApJ, 111, 426

Smart, W. M. \& Ali, A. 1940, MNRAS, 100, 560

Strom, K. M., Strom, S. E., \& Yost, J. 1971, ApJ, 165, 479

Walker, M. F. 1956, ApJS, 2, 365 